\title{
Primordial Magnetic Fields
}

\author{
KARI ENQVIST ${ }^{1}$ \\ Department of Physics and Helsinki Institute of Physics \\ P.O. Box 9, FIN-00014 University of Helsinki, Finland
}

January 3, 2018

\begin{abstract}
The explanation of the observed galactic magnetic fields may require the existence of a primordial magnetic field. Such a field may arise during the early cosmological phase transitions, or because of other particle physics related phenomena in the very early universe reviewed here. The turbulent evolution of the initial, randomly fluctuating microscopic field to a large-scale macroscopic field can be described in terms of a shell model, which provides an approximation to the complete magnetohydrodynamics. The results indicate that there is an inverse cascade of magnetic energy whereby the coherence of the magnetic field is increased by many orders of magnitude. Cosmological seed fields roughly of the order of $10^{-20} \mathrm{G}$ at the scale of protogalaxy, as required by the dynamo explanation of galactic magnetic fields, thus seem plausible.
\end{abstract}

\footnotetext{
1enqvist@pcu.helsinki.fi
} 


\section{Introduction}

Apart from the baryon number and the spectrum of energy density fluctuations, the physical processes that took place in the very early universe do not have many consequences that could still be directly detectable today. Most observables have been washed away by the thermal bath of the pre-recombination era. One possibility, which has recently received increased attention, is offered by the large-scale magnetic fields observed in a number of galaxies, in galactic halos, and in clusters of galaxies [1, 2]. The astrophysical mechanism responsible for the origin of the galactic magnetic fields is not understood. Usually one postulates a small seed field, which can then be either enhanced by the compression of the protogalaxy, and/or exponentially amplified by the turbulent fluid motion as in the dynamo theory [3]. The exciting possibility is that the seed field could be truly primordial [⿴囗 [1], in which case cosmic magnetic fields could provide direct information about the very early universe.

Early magnetic fields could then play an important role in particle cosmology by modifying the dispersion or clustering properties of various particles. One particular example is the fate of the neutrino: because of their magnetic moments, Dirac neutrinos propagating in the background of a magnetic field would be subject to a spin flip [5], so that a left-handed neutrino can be turned into a right-handed neutrino, giving rise to an extra effective neutrino degree of freedom and thereby affecting primordial nucleosynthesis. Dark matter particles could also be sensitive to the presence of a magnetic field. For instance, axions couple to magnetic fields, but perhaps surprisingly, it can be shown that despite the coupling, cold axion oscillations are not much affected by the presence of a primordial magnetic field [6].

The issue at hand is then: is it possible that primordial magnetic fields of significant strength exist? To answer this, first one has to find a mechanism in the early universe which is able to produce a large enough magnetic field. There are various proposals, a number of which are based on the early cosmological phase transitions, which are discussed in Sect 3. The second problem is to explain how the initial field, which is expected to be random as it is created by microphysics and having correlation lengths typical to microphysics, can grow up to be coherent enough at large length scales. This is a problem in magnetohydrodynamics which is discussed in Sect 4.

\section{Observation and the origin of galactic fields}

\subsection{Observing cosmic magnetic fields}

Cosmic magnetic fields can be observed indirectly both at optical and radio wavelengths (for recent reviews, see [1, 2]). Electrons moving in a magnetic field emit synchrotron radiation, and both its intensity and polarization can be used to extract 
information about the magnetic field; the radio emission is roughly proportional to $n_{e} B^{2}$, where $n_{e}$ is the electron density. However, one first needs to fix the relative magnitudes of the electron and magnetic field densities. Usually equipartition of magnetic and plasma energies is assumed, but in some cases free electron densities can be determined independently.

Information about distant magnetic fields, e.g. in clusters of galaxies, has been obtained by studying the Faraday rotation of polarized light. The method is based on the fact that the plane of polarization of linearly polarized electromagnetic wave rotates as it passes through plasma supporting a magnetic field. The rotation angle $\Delta \chi$ depends on the strength and extension of the magnetic field, the density of plasma, and on the wavelength $\lambda$ of radiation. The Faraday rotation measure (RM) out to a maximum redshift $z_{m}$ is defined as

$$
R M\left(z_{m}\right) \equiv \frac{\Delta \chi}{\Delta \lambda^{2}}=8.1 \times 10^{5} \int n_{e} B_{l}(z)(1+z)^{-2} d l(z) \mathrm{rad} \mathrm{m}^{-2}
$$

where $B_{l}(z)$ is the strength of the magnetic field along the line of sight in Gauss, $n_{e}(z)$ is in $\mathrm{cm}^{-3}$, and

$$
d l(z)=10^{-6} H_{0}^{-1}(1+z)(1+\Omega z)^{-1 / 2} d z \mathrm{Mpc}
$$

for a $\Lambda=0$ Friedmann universe, and $H_{0}$ is in $\mathrm{kms}^{-1} \mathrm{Mpc}^{-1}$ This method requires some independent information about the electron density along the line of sight and the field reversal scale. In our galaxy this may be deduced from the relative pulse delay and frequency of pulsars, but pulsars are too faint to be observed in external galaxies. Sometimes $n_{e}$ can be measured independently through X-ray bremsstrahlung, or by absorption line transitions in the object to be measured.

The observed RM is sensitive mainly to a regular magnetic field as small scale fluctuations tend to cancel out in the integral Eq. (1). The more tangled the field, the smaller RM is. To determine RM accurately one needs to measure at least three different wavelengths because the orientation of the polarization plane is ambiguous by multiples of $\pi$. The sign of RM allows to distinguish between the two opposite directions of the regular field. Magnetic field irregularities may be detected through depolarization, which tends to wipe out Faraday rotation at small wavelengths.

The Zeeman splitting of the spectral lines would provide a direct measure of the strength of the magnetic field, but the shifts are very small and this method is applicable mainly to our own galaxy. It has been used at radio frequencies to study various galactic molecular clouds.

Magnetic fields at the level of few $\mu \mathrm{G}$ have been detected in galaxies, in galactic halos, and in clusters of galaxies. Magnetic field appears to be a normal feature of spiral galaxies, where their mean field strengths range from $4 \mu \mathrm{G}$ in $\mathrm{M} 33$ to about $12 \mu \mathrm{G}$ [2]. Somewhat larger values can be found in the spiral arms. The field in spiral 
galaxies shows often either an axisymmetric or bisymmetric form following the spiral structure. It also seems that the magnetic field is not a relative newcomer but existed already some $6 \times 10^{9}$ years ago, as witnessed by the Faraday rotation observed in a high $(\mathrm{z}=0.395)$ redshift galaxy [7].

Edge-on galaxies often show vertical dust lines, indicating the existence of vertical magnetic field lines escaping from the disc. Synchrotron radiation emitting halos confirm this picture. The halo magnetic fields are associated with the outward flow of gas and dust ("galactic wind") generated by stellar activity and supernovae in the disc [1].

Faraday rotation measurements of radio sources inside and behind galactic clusters, combined with electron density estimates obtained from the X-ray spectrum, indicate that also many of them have sizeable magnetic fields. An analysis of the RM distribution of radio sources seen through or near 50 Abell clusters 8 implies a field strength of about $2 \mu \mathrm{G}\left(\mathrm{L}_{\mathrm{frs}} / 10 \mathrm{kpc}\right)^{-1 / 2} \mathrm{~h}_{50}^{-1}$, where $L_{\text {frs }}$ is the typical field reversal scale and $h_{50}$ is the Hubble parameter in units of $50 \mathrm{kms}^{-1} \mathrm{Mpc}^{-1}$; there are indications that $L_{\mathrm{frs}}$ in clusters is actually rather small, of the order of kpc. For example, a recent polarization data of a radio galaxy belonging to the Coma cluster implies [9] the existence of a core magnetic field with $B \gtrsim 8.3 h_{100}^{1 / 2} \mu \mathrm{G}$ tangled at scales less than $1 \mathrm{kpc}$. The fact that clusters show a magnetic field which has a strength comparable with the much denser interstellar medium of galaxies is somewhat surprising, but would of course be natural if the field had a truly primordial origin. Perhaps one piece of evidence pointing towards the existence of such a cosmological field is provided by the Hydra A cluster, for which the RMs have been determined continuously from within few arcseconds of the radio core out to a distance of 40", corresponding to $55 \mathrm{kpc}$. The high Faraday rotations observed [10], which show strong asymmetry between the north and south lobes of the cluster, can be explained in terms of a $6 \mu \mathrm{G}$ field coherent over the scale of $100 \mathrm{kpc}$, together with a tangled component of $\sim 30 \mu \mathrm{G}$.

The currently favoured explanation for the origin of the large scale galactic magnetic fields is the $\alpha-\omega$ dynamo [3], which through turbulence and differential rotation amplifies a small frozen-in seed field $\mathbf{B}_{0}$ to the observed $\mu \mathrm{G}$ field. An initially toroidal seed field, which is carried along on the disc of a rotating (spiral) galaxy, is locally distorted into a loop by the up- or downward stochastic drift of the gas. As the gas moves away from the plane of the disc, the pressure decreases and the gas expands; at the same time it is subject to a Coriolis force which will rotate it. The magnetic field lines, glued to the gas, will follow and thus a poloidal component perpendicular to $\mathbf{B}_{0}$ is generated. The small poloidal loops will reconnect and coalesce to produce a large scale field. This is the so-called $\alpha$-effect. Because the disc does not rotate like a rigid body, the field lines will be wrapped and the poloidal field will induce a toroidal field; this is the $\omega$-effect. Thus the dynamo mechanism does not only produce a large scale 
field but can also, to some extent, predict the shape of the field.

The dynamo saturates when the growth enters the non-linear regime. A typical growth time is of the order of $10^{9}$ years, with the rotation period of the galaxy setting a lower limit on the growth time. It should however be pointed out that there are some indications that the saturation might actually be too fast for a large-scale field to form [11]. In any cae, the strength of the required initial seed field is rather uncertain, but as a rule of thumb one could use a value like $10^{-20} \mathrm{G}$ on a comoving scale of the protogalaxy $(100 \mathrm{kpc})$.

Another possibility is that the galactic field results directly from a primordial field, which gets compressed when the protogalactic cloud collapses. The primordial field strengths needed are however quite large, of the order of $10^{-9}-10^{-10} \mathrm{G}$. In any case, it appears as if a primordial field is required to explain the observed galactic magnetic fields, although it is conceivable that a purely astrophysical solution could exist as well. For instance, within the nucleus of the "starburst galaxy" M82 there is a small but extremely active zone of star formation, which gives rise to considerable outflow of material. Associated with it is an outward trasportation of magnetic field of about $50 \mu \mathrm{G}$, which manifests itself through field lines connected with the streaming motions of the synchrotron emitting relativistic plasma [2, 12]. The field structure cannot be attributed to the action of an $\alpha-\omega$ dynamo. This demonstrates that other mechanisms exist which are able to amplify and create magnetic fields at least in the halos of galaxies. Whether purely astrophysical processes could explain galactic magnetic fields remains however to be seen, although some suggestions have been put forward. For instance, density and temperature fluctuations could create weak magnetic fields via the so-called Bierman battery mechanism which subsequently could be enhanced by turbulence [13. A more exotic suggestion is that rotating black holes could be responsible for the seed field [14]. The idea requires asymmetric particle emission from the black hole to create a current and hence a magnetic field.

\subsection{Magnetohydrodynamics in curved space}

Before discussing limits on primordial magnetic fields, it is useful to have a brief theoretical digression. The behaviour of the early magnetic field is described by the magnetohydrodynamic (MHD) equations [15]. To derive them, one assumes that the energy-momentum tensor is the sum of an ideal fluid and Maxwell parts which, in the absence of viscosity and magnetic diffusivity, reads

$$
T^{\mu \nu}=(p+\rho) U^{\mu} U^{\nu}+p g^{\mu \nu}+\frac{1}{4 \pi}\left(F^{\mu \sigma} F_{\sigma}^{\nu}-\frac{1}{4} g^{\mu \nu} F_{\lambda \sigma} F^{\lambda \sigma}\right)
$$

Here $U^{\mu}$ is the four-velocity of the plasma, normalized as $U^{\mu} U_{\mu}=-1$, and $F_{\mu \nu}=$ $\partial_{\mu} A_{\nu}-\partial_{\nu} A_{\mu}$ is the electromagnetic field tensor. Note that, as long as diffusion can 
be neglected, the presence of the magnetic field does not change the equation of state of an ideal fluid. The equations of motion for the fluid arise from energy-momentum conservation

$$
T_{; \nu}^{\mu \nu} \equiv \frac{1}{\sqrt{-g}} \frac{\partial}{\partial x^{\nu}} \sqrt{-g} T^{\mu \nu}+\Gamma_{\nu \lambda}^{\mu} T^{\nu \lambda}=0 .
$$

The Maxwell equations read

$$
F_{; \nu}^{\mu \nu}=J^{\mu}, \quad F_{[\mu \nu, \lambda]}=0 .
$$

In a flat, isotropic and homogeneous universe with a Robertson-Walker metric $d s^{2}=$ $-d t^{2}+R^{2}(t) d \mathbf{x}^{2}$, we may define $F_{\mu \nu}$ in terms of the electric and magnetic fields as

$$
F_{i 0}=R E^{i}, \quad F_{i j}=\epsilon_{i j k} R^{2} B^{k}
$$

where latin letters go from 1 to 3 . With this definition the expression for the total energy has no $R$-factors and takes therefore the familiar form

$$
T^{00}=(p+\rho) \gamma^{2}-p+\frac{1}{2}\left(\mathbf{B}^{2}+\mathbf{E}^{2}\right)
$$

where $\gamma=U^{0}$.

The MHD equations take a particularly simple form when using the conformal metric $d s^{2}=a^{2}(\tau)\left(d \tau^{2}-d \mathbf{x}^{2}\right)$. The Maxwell equations can then be written as

$$
\frac{\partial \tilde{\mathbf{B}}}{\partial \tilde{t}}=-\nabla \times \tilde{\mathbf{E}}, \quad \nabla \cdot \tilde{\mathbf{B}}=0
$$

and

$$
\tilde{\mathbf{J}}=\boldsymbol{\nabla} \times \tilde{\mathbf{B}}-\frac{\partial \tilde{\mathbf{E}}}{\partial \tilde{t}}, \quad \nabla \cdot \tilde{\mathbf{E}}=\tilde{\rho}_{e}
$$

where the scaled variables are defined as

$$
\tilde{\rho}_{e}=a^{4} \rho_{e}, \quad \tilde{\mathbf{B}}=a^{2} \mathbf{B}, \quad \tilde{\mathbf{J}}=a^{3} \mathbf{J}, \quad \text { and } \quad \tilde{\mathbf{E}}=a^{2} \mathbf{E} .
$$

Here $\rho_{e}$ is the charge density.

The MHD equations in curved space, using conformal metric, thus have a similar form to those in flat space. Let us therefore consider the flat space for simplicity. The evolution of the magnetic field is described by

$$
\frac{\partial \mathbf{B}}{\partial t}=\nabla \times(\mathbf{v} \times \mathbf{B})-\nabla \times \frac{1}{\sigma}(\nabla \times \mathbf{B})
$$

which follows from the Maxwell equations Eqs. (8,9) and Ohm's law. In a perfect conductor $\sigma=\infty$ and field lines are frozen in to the fluid. If $\sigma$ is finite, the fluid can slip through the field lines. The quantitative measure of the freezing is given by the 
magnetic Reynolds number $R_{M}$. It can be defined through the ratio of the two terms in Eq. (11) as

$$
R_{M} \simeq \frac{\sigma|\nabla \times(\mathbf{v} \times \mathbf{B})|}{\left|\nabla^{2} \mathbf{B}\right|} \sim \sigma v L
$$

where $L$ is the length scale and $v$ is the bulk or drift velocity of the fluid. If $R_{M} \gg 1$, the magnetic flux lines will be frozen into the plasma. In the astrophysical context the

fluid can often be considered as an extremely good conductor with $R_{M} \gg 1$. The early universe is also a good conductor [16, 17] with $\sigma \sim T$ and $L \sim M_{P l} / T^{2}$ so that easily $R_{M} \gg 10^{10}$. Large $R_{M}$ signals the onset of turbulence, which thus could be expected to play a role in the early universe magnetohydrodynamics, as will be discussed in Sect. 4.

\subsection{Cosmologial fields: limits and observations}

Observing cosmological, intergalactic magnetic fields would naturally be of great importance as it would strengthen the case for their truly primordial origin. While the fields in the neighbourhood of galaxies or clusters of galaxies could conceivably originate in the ejected magnetoplasma, magnetic fields in the voids, which are devoid of baryonic matter, would be much harder to explain in terms of conventional astrophysics.

Energetic $\gamma$-rays can traverse the intergalactic voids, and it has been claimed by Plaga [18] that the arrival times of extragalactic $\gamma$-rays could be used to detect fields as weak as $10^{-24} \mathrm{G}$. The idea is that cosmic rays, originating from far-away objects such as QSOs or gamma-ray bursters, scatter off the background cosmic magnetic field. This gives rise to pair production and a delayed $\gamma$-ray which could then be observed, and the ratio of prompt to delayed $\gamma$ 's provides a measure of the strength of the intergalactic magnetic field. The energy spectrum of $\gamma$-rays is likewise affected by the intergalactic magnetic field [19]. Ultra-high cosmic ray protons would also be deflected by the intergalactic magnetic fields so that their arrival times could be used [20] to set bounds as low as $10^{-12} \mathrm{G}$.

A constraint on the strength of a cosmological magnetic field has been obtained by considering the rotation measure of a sample of 309 galaxies and quasars, which yields the limit [21] $B \leq 10^{-9} \mathrm{G}\left(\Omega_{\mathrm{IG}} \mathrm{h}_{100} / 0.01\right)^{-1}$, where $\Omega_{I G}$ is the fraction of the ionized gas density of the critical density in the intergalactic medium. The assumption here is that the field is coherent over the horizon scale, and the limit is weakened if the field is tangled at smaller scales, as in fact would seem likely. It has also been suggested that the power spectrum of the cosmological field could be determined by studying the correlations in the RM for extragalactic sources [22].

If a primordial magnetic field is present at the time of recombination, it will give rise to anisotropic pressures. These would distort the microwave background. Barrow, 
Ferreira and Silk 23 have recently considered the effect of a magnetic field on the evolution of shear anisotropy $\sigma_{A}$ in a general anistropic flat universe. For a fluid with an equation of state given by $p=(\gamma-1) \rho$ (with $0<\gamma \leq 4 / 3)$ it was found that

$$
\frac{\sigma_{A}}{H}=\frac{4}{2-\gamma}\left(\frac{\rho_{B}}{\rho}+\frac{\rho_{g a}}{\rho}\right)+\delta t^{(\gamma-2) / \gamma},
$$

where $\rho_{g a}$ is the gas anistropy, $\rho_{B}=B^{2} / 8 \pi$ is the magnetic energy density, and $\delta$ is a constant. The angular anisotropy $\Delta T$ is then directly proportional to $\sigma_{A} / H$. Barrow, Ferreira and Silk then compared the result with the 4-year COBE data set, and assuming that the whole observed anisotropy is due to magnetic stresses only, concluded that, if the field can be taken homogeneous, $B \leq 3.4 \times 10^{-9} \mathrm{G}\left(\Omega_{0} \mathrm{~h}_{50}^{2}\right)^{1 / 2}$. This is a more stringent limit than what can be obtained [24, 25, 26] from primordial nucleosynthesis considerations.

The anisotropy limit also indicates that the distortions of the Doppler peaks of the microwave background [27] due to a homogeneous magnetic field are unobservable, although distortions due to an inhomogeneous magnetic field may still be observable. The distortions arise because in the presence of a magnetic field the wave patterns generating fluctuations in the energy density change. There are magnetosonic waves, which in the absence of a magnetic field would correspond to sound waves, together with Alfvén waves propagating with velocity $v_{A}$, which however do not induce energy density fluctuations but affect the velocities of baryons and photons [27]. The fast magnetosonic waves effectively change the sound velocity $v_{S}$ in the baryon fluid by $v_{S}^{2} \rightarrow v_{S}^{2}+v_{A}^{2} \sin ^{2} \theta$, where $\theta$ is the angle between $\mathbf{B}$ and the wave vector $\mathbf{k}$, thereby changing the Doppler peak structure.

The Faraday rotation in the polarization of the microwave background could still be observable, as has been argued by Kosowsky and Loeb [28]. They estimate that for a primordial field corresponding to the present value of $10^{-9} \mathrm{G}$, coherent on the scale of the last scattering surface, there should be a rotation angle of $1^{\circ}$ at $30 \mathrm{GHz}$. Such a signal requires a beam size of order $10^{\prime}$ and could conceivably be detected in the future microwave background maps.

\subsection{Limits from primordial nucleosynthesis}

Primordial nucleosynthesis offers a laboratory for many particle physics ideas. It also provides a testing gorund for primordial magnetic fields, which would modify the calculated element abundances through two effects [24, 26]. The extra magnetic field energy density would accelerate the expansion rate of the universe, and hence increase the predicted abundance of ${ }^{4} \mathrm{He}$. If one defines the magnetic field at the end of nucleosynthesis $\left(T=10^{9} \mathrm{~K}\right)$ in terms of the critical field $B_{c} \equiv e B / m_{e}^{2}=4.4 \times 10^{13} \mathrm{G}$ with $B=\gamma B_{c}$, one finds numerically [26] that $\gamma=3 \times 10^{-3}$ is sufficient to push 
the relative ${ }^{4} \mathrm{He}$-abundance above 0.24 . There is also a small effect on the ${ }^{7} \mathrm{Li}$ and $\left(\mathrm{D}+{ }^{3} \mathrm{He}\right)$ abundances, but these are negligible.

However, magnetic fields affect the Hubble rate yet in another, more subtle way [24, 26]. The statistical distributions of relativistic electrons and positrons change because of the background magnetic field. The particles are no longer plane waves but occupy Landau levels, with the dispersion relation given by (for $B$ along z-axis)

$$
E=\left(p_{z}^{2}+m_{e}^{2}+2 e B n_{s}\right)^{1 / 2}+m_{e} \kappa
$$

where $n_{s}=n+1 / 2-s_{z}$ with $s_{z}= \pm 1 / 2$ and $n$ the Landau level, and $\kappa$ is the anomalous magnetic moment term, which for fields less than about $10^{16} \mathrm{G}$ reads $\kappa=e \alpha B /\left(4 \pi m_{e}^{2}\right)$; for subcritical fields, its effect is small. The number density of states in the interval $d p_{z}$ is then

$$
\left(2-\delta_{n_{s}, 0}\right) \frac{e B}{(2 \pi)^{2}} d p_{z} .
$$

If $e B \ll T^{2}$, as is natural, a fraction of the electron-positron pairs condense in the lowest Landau level. As a consequence, the number and energy densities of electrons and positrons increase with respect to the zero field case.

A primodial magnetic field affects primordial nucleosynthesis also by increasing $n \leftrightarrow p$ reaction rates [24, 26]. Hence ${ }^{4} \mathrm{He}$ abundance tends to decrease, partly counteracting the effect due to change in the Hubble expansion rate. It turns out, however, that this effect is also small. More important is the modification of the phase space of the electrons: increasing number and energy densities causes a decrease in all of the weak interaction rates. Taking all these effects into account and requiring that the ${ }^{4} \mathrm{He}$ abundace should not exceed 0.245, Grasso and Rubinstein obtain [26] the upper limit

$$
B \leq 1 \times 10^{11} \mathrm{G}
$$

at $T=10^{9} \mathrm{~K}$. A similar conclusion has also been obtained in [24]. Here the magnetic field was assumed to be uniform; if inhomogeneities on the scale much less than the horizon are present, as seems likely, the effect of the magnetic field on weak rates could be averaged out. In this case Grasso and Rubinstein obtain the limit

$$
B \leq 1 \times 10^{12} \mathrm{G}
$$

This assumes a magnetic field coherence length $L_{0}$ in the range $10 \ll L_{0} \ll 10^{11} \mathrm{~cm}$.

\section{Generating primordial magnetic fields}




\subsection{Early phase transitions}

There are a number of proposals for the origin of magnetic fields in the early universe. Fluctuations in the electromagnetic field in a relativistic plasma are by themselves sufficient for generating a small scale random magnetic field [29]. To obtain a seed field for galactic magnetic fields, one however needs fields with much larger coherence lengths. A natural place to look for large scale magnetic fields would be the early cosmological phase transitions, such as the electroweak or the QCD phase transition.

Magnetic fields may arise in a electrically neutral plasma if local charge separation happens to take place, thus creating a local current. It has been proposed that this could occur during a first order QCD [30] or EW [31, 32] phase transition, which proceed by nucleating bubbles of the new phase in the background of the old phase. There one finds net baryon number gradients at the phase boundaries, providing the basis for charge separation, and the seed fields arise through instabilities in the fluid flow. For that one has to require that the growing modes are not damped. Turbulent flow near the walls of the bubbles is then expected to amplify and freeze the transient seed field. The various hydrodynamical features have been studied in linear perturbation theory by Sigl, Olinto and Jedamzik [32], who argued that on a $10 \mathrm{Mpc}$ comoving scale, field strengths of the order of $10^{-29} \mathrm{G}$ for EW and $10^{-20} \mathrm{G}$ for QCD could be obtained. These might further be enhanced by several orders of magnitude by hydromagnetic turbulence [33, 34], which will be discussed in Section 4.3.

\subsection{Bubble collisions}

In a first order phase transition the phases of the complex order parameter $\Phi=$ $\rho e^{i \Theta} / \sqrt{2}$ of the nucleated bubbles are uncorrelated. When the bubbles collide, there arises a phase gradient which acts as a source for gauge fields. The phase itself is not a gauge invariant concept, but it has been pointed out by Kibble and Vilenkin [35] that a gauge invariant phase difference can be defined in terms of an integral over the gradient $D_{\mu} \Theta$.

Magnetic field generation in the collision of phase transition bubbles has been considered in the abelian Higgs model [35, 36]. One assumes that inside the bubble the radial mode $\rho$ settles rapidly to its equilibrium value $\eta$ and can thus be treated as a constant. The dynamical variables are thus $\Theta$ and the gauge field $A^{\mu}$. The starting point is the $\mathrm{U}(1)$-symmetric lagrangian

$$
\mathcal{L}=-\frac{1}{4} F_{\mu \nu} F^{\mu \nu}+D_{\mu} \Phi\left(D^{\mu} \Phi\right)^{\dagger}+V(|\Phi|),
$$

where the potential $V$ is assumed to have minima at $\rho=0$ and $\rho=\eta$. The simplest case is that two spherical bubbles nucleate, one at $(x, y, z, t)=\left(0,0, z_{0}, 0\right)$ and the other at $(x, y, z, t)=\left(0,0,-z_{0}, t_{0}\right)$, and keep expanding with the velocity $v$ even after 
colliding. Because of the symmetry of the problem, the solutions to the equations of motion are functions of $z$ and $\tau=\sqrt{t^{2}-x^{2}-y^{2}}$ only, and the $\mathrm{U}(1)$ gauge field $A^{\mu}=x^{\mu} f(\tau, z)$. One finds [35 that the generated magnetic field is rapidly oscillating and orthogonal to the z-axis and in this case confined inside the intersection region. It has a ring-like shape in the $(\mathrm{x}, \mathrm{y})$-plane. It can also be shown that subsequent collisions of the bubbles, which now may have a magnetic field inside the bubbles, nevertheless lead to a qualitatively similar outcome [36].

There are however two additional important ingredients which need to be taken into account: the high but finite conductivity [16, 17] of the primordial plasma and the fact that in the electroweak phase transition the bubbles will in fact intersect with nonrelativistic velocities [37]. Finite conductivity gives rise to diffusion, the consequence of which is to smooth out the rapid oscillations of $\mathbf{B}$, whereas low $v$ will permit the magnetic flux to escape the intersection region and penetrate the colliding bubbles, where its evolution will be governed by usual magnetohydrodynamics.

The strength of the generated magnetic field depends on the bubble wall velocity in an essential way [36]. In the electroweak case the initial growth of the bubble wall is by subsonic deflagration, with velocities of the order of $0.05 c$, depending on the assumed friction strength [37]. The wall is preceded by a shock front, which may collide with the other bubbles. This results in reheating, and oscillations of the bubble radii, but eventually a phase equilibrium is attained. The ensuing bubble growth is very slow and takes place because of the expansion of the universe. Because the universe has been reheated back to $T_{c}$, no new bubbles are formed during the slow growth phase. Assuming that the abelian Higgs model results are applicable to the electroweak case, one may estimate that [36]

$$
B \simeq 2.0 \times 10^{20} \sqrt{\gamma^{2}+2 \gamma R} / R \mathrm{G}
$$

where it has been assumed $\Theta_{0}=1, T_{c}=e \eta=100 \mathrm{GeV}$; the average distance between the nucleation centers $r_{\text {ave }}=9.5 \times 10^{-8} t_{H}$ and the velocity $v=1.2 \times 10^{-4}$ were taken as reference values. Folding in the spectrum of separation of the adjacent shocked spherical bubbles [40, averaging over all possible inclinations of the ring-like magnetic field, and taking into account the enhancement of magnetic energy due to an inverse cascade (see next Section), one arrives at the estimate $B_{\text {rms }} \simeq 10^{-21} \mathrm{G}$ for the cosmological magnetic field at the scale of $10 \mathrm{Mpc}$ today [36].

There are some indications that the behaviour of the abelian Higgs model does not differ qualitatively from the full $S U(2) \times U(1)$ case [38, 39]. However, it has also been argued by Törnkvist [41] that in the Standard Model the non-abelian nature of the gauge fields in fact forbids the formation of magnetic fields in two-bubble collisions, which in effect can be gauge rotated away. The result is valid for a specific intial bubble configuration and applies only for the initial evolution. The question is thus 
still open, but it seems likely that in bubble collisions magnetic fields are generated sooner or later. For example, in a collision of three bubbles magnetic field can no longer be rotated away [41.

\subsection{Fluctuating Higgs gradients}

It has been pointed out by Vachaspati [42] that fluctuating Higgs field gradients will induce a magnetic field. Such local fluctuations are naturally present at the EW phase transition, no matter what its order, since the embedding of the electromagnetic field in $S U(2) \otimes U(1)_{Y}$ involves these gradients:

$$
F_{i j}^{e m}=-i\left(V_{i}^{\dagger} V_{j}-V_{i} V_{j}^{\dagger}\right), \quad V_{i}=\frac{2}{|\phi|} \sqrt{\frac{\sin \theta}{g}} \partial_{i} \phi,
$$

where $\phi$ is the Higgs field. At the electroweak phase transition the field strength $F_{i j}^{e m}$ can be expected to be constant and $\mathcal{O}\left(m_{W}\right)^{2}$ but varies in a random way over larger distances. The vector $V_{i}$ is also random, of course. Its variation is due to the fact that the Higgs field $\phi$ makes a random walk on the vacuum manifold of $\phi$.

To find out the root-mean-square field at large distance scales, one should make use of a proper statistical argument. The question then is, what sort of an average should one use? The line average, where the gradient vectors are taken to be the basic stochastic variables [44, gives rise to a scaling behavior $B \sim 1 / \sqrt{L}$. Because measuring the strength of $B$ by Faraday rotation of the emitted light involves the average along the line of sight, this averaging method could indeed be appropriate in these cases. It has however been argued that for the galactic dynamo, only the volume average has dynamical significance [45]. This has been shown to imply that, in the absence of any turbulent enhancement, even under the most favourable circumstances the electroweak phase transition can manage only about $10^{-20} \mathrm{G}$ on the $1 \mathrm{Mpc}$ scale [45].

\subsection{Vacuum condensates}

Another, a more exotic possibility for generating primordial magnetic fields is based on the observation that, due to quantum fluctuations, the Yang-Mills vacuum is unstable in a large enough background magnetic field [46] at zero temperature.

In the early universe the effective energy picks up thermal corrections from the fermion, gauge boson, and Higgs boson loops. The detailed form of the thermal correction depends on the actual model, but one may take the cue from the $\mathrm{SU}(2)$ one-loop calculation, where they are obtained by summing the Boltzmann factors $\exp \left(-\beta E_{n}\right)$ for the oscillator modes

$$
E_{n}^{2}=p^{2}+2 g B\left(n+\frac{1}{2}\right)+2 g B S_{3}+m^{2}(T),
$$


where $S_{3}= \pm 1 / 2( \pm 1)$ for fermions (vectors bosons). Eq. (21) includes the thermally induced mass $m(T) \sim g T$, corresponding to a ring summation of the relevant diagrams. Numerically, the effect of the thermal mass turns out to be very important. At high temperature, the leading behaviour is given mainly by the bosonic contributions, and thus one arrives at the estimate 47]

$$
\delta V_{T}^{v}=\frac{(g B)^{2}}{8 \pi^{2}} \sum_{l=1}^{\infty} \int_{0}^{\infty} \frac{d x}{x^{3}} e^{-K_{l}^{b}(x)}\left[x \frac{\cosh (2 x)}{\sinh (x)}-1\right],
$$

where $K_{l}^{a}(x)=g B l^{2} /\left(4 x T^{2}\right)+m_{a}^{2} x /(g B)$ and numerically $\delta V_{T}^{v} \sim 0.02(g B)^{2}$ which serves only to shift the value of $B$ at the vacuum slightly. Thus $B \neq 0$ is the state of the lowest energy even at high temperature.

A local fluctuation will then trigger the creation of the a new vacuum with nonzero non-abelian magnetic field inside a given particle horizon at scales $\mu \simeq T$. The Maxwell magnetic field is then just a projection of the non-abelian field. The resulting magnetic field is exponentially suppressed and can safely be neglected in the case of the electroweak gauge group. In the early universe, however, where possibly a grand unified symmetry is valid, the suppression may be less severe 477. It is also attenuated by the running of the coupling constant. One can then estimate that

$$
B(T)=g_{\mathrm{GUT}}^{-1} \mu^{2} \exp \left(-\frac{48 \pi^{2}}{11 N g^{2}}\right)\left(\frac{T^{2}}{\mu^{2}}\right) \simeq 3 \times 10^{42}\left(\frac{a\left(t_{\mathrm{GUT}}\right)}{a(t)}\right)^{2} \mathrm{G},
$$

where the reference number is for susy $\mathrm{SU}(5)$. The field is large enough to serve as the seed field.

Electroweak magnetic condensates have also recently been considered by Cornwall [48], who has suggested that they would give rise to magnetic fields with a net helicity via the generation of electroweak Chern-Simons number. Joyce and Shaposnikov 49. have argued that a right-handed electron asymmetry, generated at the GUT scale, could give rise to a hypercharge magnetic field $B_{Y}$ via a Chern-Simons term. $B_{Y}$ is coupled to the baryonic current via the anomaly, and would thus induce baryon number fluctations which could survive until primordial nucleosynthesis [50], making it inhomogenous. These considerations deserve further study.

\subsection{Structure formation and and magnetic fields}

A natural idea is that primordial fields are induced by the mechanism responsible for the structure formation, such as inflation. However, the basic problem with inflation with regards to magnetic field generation is that the early universe is a good conductor so that, ignoring turbulence, the magnetic flux $\sim B a^{2}$ tends to be conserved. To avoid this, one needs to break the conformal invariance somehow, as was first suggested by Turner and Widrow [16], who considered couplings to the curvature $R$ such as 
$R F^{2}$ and $R A^{2}$, as well as photon-axion couplings. Dilaton coupling of the form $e^{\Phi} F^{2}$ has also been considered [51], and interesting field strengths can be obtained at the expense of tuning the dilaton coupling strength. If a phase transition takes place during the inflationary period, a sufficiently large magnetic field could be created, provided however that the phase transition takes place during the final 5 e-foldings [52].

Cosmic strings have also been suggested to be responsible for magnetic fields [53], the field being generated by the vortical motion inside the wakes of strings. There are however some open questions related to the stability of the strings, which might be remedied if the strings are superconducting [54]. There also exist speculations [55] about superstringy origin of the magnetic field.

\section{From microscopic to macroscopic fields}

\subsection{Magnetohydrodynamic turbulence}

Even assuming that a primordial magnetic field is created at some very early epoch, a number of issues remain to be worked out before one can say anything definite about the role primordial fields in the formation of galactic magnetic fields. At the earliest times magnetic fields are generated by particle physics processes with length scales typical to particle physics. (It has been shown that such fields are stable against thermal fluctuations [56].) The remaining question is whether it is at all possible for the small scale fluctuations to grow to large scales, and what exactly is the scaling behaviour of $B_{\mathrm{rms}}$ or the correlator $\langle B(r+x) B(x)\rangle$. To study these problems one needs to consider the detailed evolution of the magnetic field to account for such issues as to what happens when uncorrelated field regions come into contact with each other during the course of the expansion of the universe. In general, turbulence is an essential feature of such phenomena. These questions can only be answered by considering MHD in an expanding universe [15], but a big problem is the very large Reynolds number of the early universe. Realistic simulations are simply not possible. However, simulations can nevertheless be useful in giving an indication of the possible trend. Starting with a random $2 \mathrm{~d}$ magnetic field configuration in a radiation dominated FRW-universe, one finds that the magnetic structures coalesce and there is a gradual emergence of larger and larger magnetic scales [33]. Such a behaviour is encouraging, indicating that the field is not really comovingly frozen. On the other hand, because of the different topology, 2d MHD is very different from the 3d MHD, and moreover, the Reynolds number in the simulation (about 10) is still quite unrealistic.

Dimopoulos and Davis [57 have also argued out that when two initially uncorrelated domains come into contact, the field at the interface should untangle with the 
plasma bulk velocity $v$ to avoid the creation of domain walls. They propose that the correlation length $\xi$ evolves according to

$$
\frac{d \xi}{d t}=H \xi+v
$$

where $H$ is the Hubble parameter and the velocity $v$ depends dynamically on $B$ and should, in principle, be determined from MHD. Qualitatively, this argument also points towards a field not frozen in the plasma.

\subsection{Shell models}

Large $R_{M}$ raises the possibility of magnetohydrodynamic turbulence, or effects not visible in local perturbation theory. In ordinary hydrodynamics many properties of turbulence, in particular those related to energy transfer and to the spectral properties have been studied successfully using a simple cascade model. This is true not only qualitatively, but also quantitatively, which is the reason why the cascade model is now much used in nonlinear physics [58].

The basic idea is that the interactions due to the nonlinear terms in the MHD equations are local in wavenumber space, and in $k$-space the quadratic nonlinear terms become a convolution. Interactions in $k$-space involving triangles with similar side lengths have the largest contribution. This has led to the shell model which is formulated in the space of the modulus of the wave numbers. This space is approximated by $\mathrm{N}$ shells, where each shell consists of wave numbers with $2^{n} \leq k \leq 2^{n+1}$ (in the appro-

priate units). The Fourier transform of the velocity over a length scale $k_{n}^{-1}\left(k_{n}=2^{n}\right)$ is given by the complex quantity $v_{n}$, and $B_{n}$ denotes a similar quantity for the $B$-field. Furthermore, the convolution is approximated by a sum over the nearest and the next nearest neighbours,

$$
N_{n}(v, B)=\sum_{i, j=-2}^{2} C_{i j} v_{n+i} B_{n+j} .
$$

Here $v$ and $B$ have lost their vectorial character, which reflects the fact that this model is not supposed to be an approximation of the original equations, but should be considered as a toy model that has similar conservation properties as the original equations.

Velocity and magnetic fields are thus represented by scalars at the discrete wave numbers $k_{n}=2^{n}(n=1, \ldots, N)$, i.e. $k_{n}$ increases exponentially. Therefore such a model can cover a large range of length scales (typically up to ten orders of magnitude). The important conserved quantity is $E_{\mathrm{tot}} a^{4}$, where $E_{\text {tot }}=\int T^{00} d^{3} x$ is the total energy. The resulting equations of motion read, in conformal time $\tilde{t}, 33$

$$
\frac{4}{3} \rho_{0} \frac{d v_{n}}{d \tilde{t}}=N_{n}(v, b)
$$



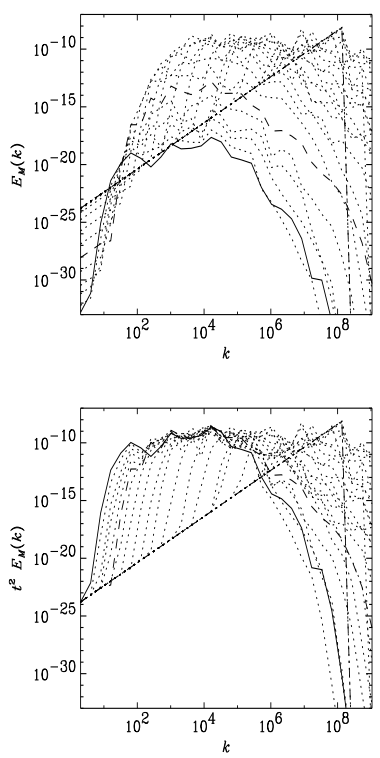

Figure 1: Spectra of the magnetic energy at different times. The straight dotteddashed line gives the initial condition $\left(t_{0}=1\right)$, the solid line gives the final time $\left(t=3 \times 10^{4}\right)$, and the dotted curves are for intermediate times (in uniform intervals of $\left.\Delta \log \left(t-t_{0}\right)=0.6\right)$.

$$
\frac{d b_{n}}{d \tilde{t}}=M_{n}(v, b)
$$

where $\rho_{0}$ is the background radiation density, and $\mathbf{b}=\mathbf{B} a^{2}$. The functions $N_{n}$ and $M_{n}$ each have three terms (combinations of $v_{j}$ and $b_{j}$ ), the form of which is determined by the MHD equations, with three free parameters $A, B$, and $C$ [33].

\subsection{Inverse cascade}

The numerical study of the cascade model requires of course that the parameters $A, B, C$ are fixed so that the model has the same conservation laws as the full-fledged MHD. One ignores the detailed evolution of the density by setting $\rho \simeq \rho_{0} a^{-4}$ so that

$$
\int\left(\frac{4}{3} \rho_{0} \mathbf{v}^{2}+\frac{1}{2} \mathbf{b}^{2}\right)=\text { const. }
$$

The model can then be solved numerically [33], and the results are shown in Fig. 1 , where the transfer of magnetic energy to larger and larger length scales is clearly seen. This process, the inverse cascade, is due to the nonlinear terms giving rise to mode interactions. The initial magnetic energy spectrum was chosen to be given by $E_{M}(t=0) \sim k$ with the total magnetic energy equal to $\rho_{0}$. The number of shells was $N=30$ so that length scales differing by ten orders of magnitude were covered. It was found that the integral scale, which measures where most magnetic energy is concentrated and which is given by

$$
l_{0}=\int(2 \pi / k) E_{M}(k) d k / \int E_{M}(k) d k
$$



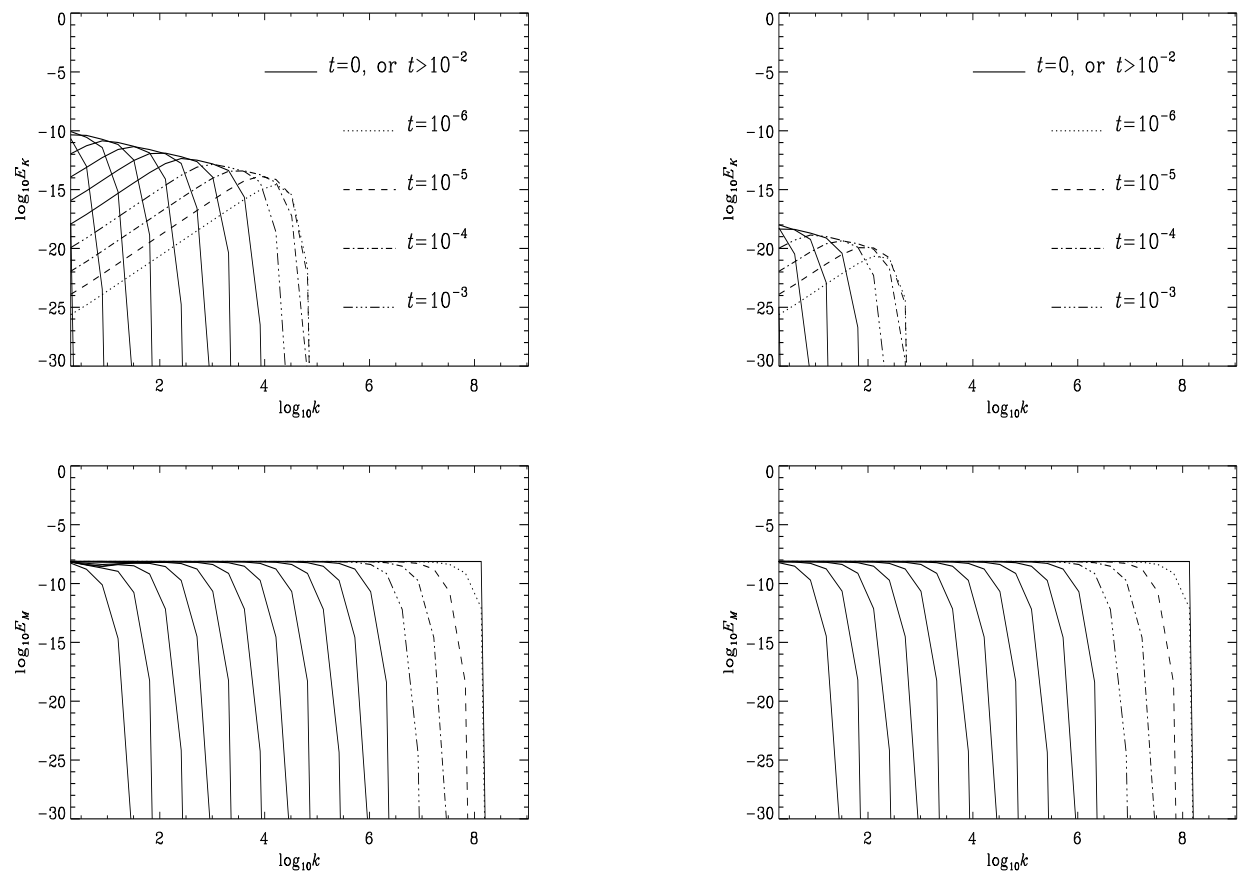

Figure 2: Magnetic and plasma kinetic energy spectrum as a function of the wave number $k$ in the cascade model for small $\left(\nu=10^{-2}\right)$ plasma viscosity (left) and large $\left(\nu=10^{2}\right)$ plasma viscosity (right). The initial spectrum has been taken to be flat, in accordance with the large-time behaviour of Fig. 1. The highest time $t=10^{8}$ corresponds to the Hubble time $10^{16}$.

where $E_{M}(k)$ is the magnetic energy spectrum, increases with the Hubble time approximately like $t_{H}^{0.25}$.

However, around the time of recombination the photon mean free path $\lambda_{\gamma}$ is very large and photon diffusion becomes very efficient in smoothing out virtually all inhomogeneities of the photon-baryon plasma [59]. This process is often referred to as Silk damping, which corresponds to a kinematic viscosity $\nu \simeq \lambda_{\gamma}$ (in natural units). Silk damping may thus destroy the magnetic field, as has been noted by Jedamzik, Katalinic and Olinto [60. Therefore one has to follow numerically the evolution of the magnetic and kinetic energy spectra in the presence of kinematic viscosity. The results [34] are presented in Fig. 2 and the main point can be summarized as follows: in the cascade models magnetic energy is transferred to large length scales even in the presence of large viscosity. Here the initial magnetic spectrum was chosen to be flat in accordance with the large time behaviour suggested by Fig. 1.

For a sufficiently large viscosity, the inverse cascade stops. One may estimate [34 that this typically takes place close to recombination. The results suggest that in the real MHD, inverse cascade is operative and is essentially not affected by Silk damping, except very late and perhaps for very weak fields. Thus we may conclude that it is unlikely that an equipartition exists in the very early universe. A similar conclusion 
can be drawn in a different, continuous model where the inverse cascade can be found analytically in an appropriate scaling regime [34, 61].

\section{Conclusions}

Explaining the galactic magnetic fields in terms of microphysical processes that took place when the universe was only ten billionth of a second old is a daunting task, which is not made easier by the complicated evolution of the magnetic field as it is twisted and tangled by the flow of plasma. It is nevertheless encouraging that mechanisms for generating primordial magnetic fields of suitable size exist, and in particular those based on the early cosmological phase transitions discussed in Sect. 3 look promising. At the same time the fact that there are so many possibilities tends to underline our ignorance of the details of the subsequent evolution of the magnetic field. The step from microphysics to macroscopic fields is a difficult one because of the very large magnetic Reynolds number of the early universe. However, different considerations, both analytic approximations, $2 \mathrm{~d}$ simulations, as well as the full-fledged shell model computations which can account for turbulence, seem to point to the existence of an inverse cascade of magnetic energy. Moreover, as discussed in Sect. 4.3, the inverse cascade is obtained also in the presence of a large plasma viscosity. Therefore the primordial origin of the galactig magnetic fields is quite possible.

Much theoretical work remains to be done, though. At the same time it is very important that progress is made on the observational front. In particular, measuring or setting a stringent limit on the intergalactic field, which could be possible in the near future as indicated in Sect. 2.3, would provide the testing ground for all theoretical scenarios.

\section{Acknowledgments}

I wish to thank Poul Olesen for a fruitful collaboration on primordial magnetic fields, and Ola Törnkvist for useful discussions on bubble collisions. This work has been supported by the Academy of Finland. 


\section{References}

[1] P.P. Kronberg, Rep. Prog. Phys. 57 (1994) 325.

[2] R. Beck et. al., Ann. Rev. Astron. Astrophys. 34 (1996) 153.

[3] L. D. Landau and E.M. Lifshitz, Electrodynamics of Continuous Media (Pergamon, Oxford 1960); Ya.B. Zeldovich, A.A. Ruzmaikin and D.D. Sokoloff, Magnetic Fields in Astrophysics (McGraw-Hill, New York, 1980); E.N. Parker, Cosmological Magnetic Fields (Oxford Univ. Press, Oxford, 1979); A.A. Ruzmaikin, A.A. Shukurov and D.D. Sokoloff, Magnetic Fields of Galaxies (Kluwer, Dordrecht, 1988); E. Battaner, Astrophysical Fluid Dynamics (Cambridge University Press, Great Britain, 1996).

[4] For a discussion of the astrophysical issues involved, see. e.g. A. M. Howard and R. M. Kulsrud, astro-ph/9609031.

[5] K. Enqvist, A. I. Rez and V. B. Semikoz, Nucl. Phys. B436 (1995) 49; P. Elmfors, D. Grasso and G. Raffelt, Nucl. Phys. B479 (1996) 3; P. Elmfors, K. Enqvist, G. Raffelt and G. Sigl, Nucl. Phys. B503 (1997) 3.

[6] J.T. Ahonen, K. Enqvist and G. Raffelt, Phys. Lett. B366 (1996) 224.

[7] P.P. Kronberg, J.J. Perry and E.L.H. Zukovski, Ap. J. 387 (1992) 528.

[8] K.-T. Kim, P.P. Kronberg and P.C. Tribble, Ap. J. 379 (1991) 80.

[9] L. Feretti et al., Astron. Astrophys. 302 (1995) 680.

[10] G.B. Taylor and R.A. Perley, Ap. J. 416 (1993) 554.

[11] S.I.Vainshtein, E. N. Parker and R. Rosner, Ap. J. 404 (1993) 773; F. Cattaneo, Ap. J. 434 (1994) 200.

[12] H.-P. Reuter et al., Astron. Astrophys. 282 (1994) 724.

[13] R. M. Kulsrud and S. W. Anderson, Ap. J. 396 (1992) 606; R. M. Kulsrud, R. Cen, J.P. Ostriker and D. Ryu, astro-ph/9607141.

[14] A. Vilenkin and D.A. Leahy, Ap. J. 254 (1982) 77.

[15] See e.g. R. M. Gailis, N. E. Frankel and C. P. Dettmann, Phys. Rev. D52 (1995) 6901, and references therein.

[16] M.S. Turner and L.M. Widrow, Phys. Rev. D37 (1988) 2743. 
[17] J.T. Ahonen and K. Enqvist, Phys. Lett. B382 (1996) 40; G. Baym and H. Heiselberg, astro-ph/9704214; J.T. Ahonen, hep-ph/9801434.

[18] R. Plaga, Nature 374 (1995) 430.

[19] S. Lee, A. V. Olinto and G. Sigl, Ap. J. 455 (1995) L21.

[20] M. Lemoine, G. Sigl, A. V. Olinto and D. N. Schramm, Ap. J. 486 (1997) L115.

[21] J.P. Vallée, Ap. J. 360 (1990) 1.

[22] T. Kolatt, astro-ph/9704243.

[23] J.D. Barrow, P. Ferreira and J. Silk, Phys. Rev. Lett. 78 (1997) 3610.

[24] B.-l. Cheng A. V. Olinto, D. N. Schramm and J. W. Truran, Phys. Rev. D54 (1996) 4714.

[25] P.J. Kernan, G.D. Starkman and T. Vachaspati, Phys. Rev. D54 (1996) 7207.

[26] D. Grasso and H. Rubinstein, Phys. Lett. B379 (1996) 73.

[27] J. Adams, U.H. Danielsson, D. Grasso and H. Rubinstein, Phys. Lett. B388 (1996) 253.

[28] A. Kosowsky and A. Loeb, Ap. J. 469 (1996) 1.

[29] D. Lemoine, Phys. Rev. D51 (1995) 2677.

[30] B. Cheng and A.V. Olinto, Phys. Rev. D50 (1994) 2421.

[31] G. Baym, D. Bdeker and L. McLerran, Phys. Rev. D53 (1996) 662.

[32] G. Sigl, A.V. Olinto and K. Jedamzik, Phys. Rev. D55 (1997) 4582.

[33] A. Brandenburg, K. Enqvist and P. Olesen, Phys. Rev. D54 (1996) 1291.

[34] A. Brandenburg, K. Enqvist and P. Olesen, Phys. Lett. B391 (1997) 395.

[35] T. W. B. Kibble and A. Vilenkin, Phys. Rev. D52 (1995) 679.

[36] J. T. Ahonen and K. Enqvist, Phys. Rev. D57 (1998) 664.

[37] For a recent numerical study, see H. Kurki-Suonio and M. Laine, Phys. Rev. Lett. 77 (1996) 3951.

[38] E.J. Copeland and P.M. Saffin, Phys. Rev. D54 (1996) 6088.

[39] D. Grasso and A. Riotto, hep-ph/9707265. 
[40] B.S. Meyr, C.R. Alcock and G.J. Mathews, Phys. Rev. D43 (1991) 1079.

[41] O. Törnkvist, hep-ph/9707513.

[42] T. Vachaspati, Phys. Lett. B265 (1991) 258.

[43] S. Davidson, Phys. Lett. B380 (1996) 253.

[44] K. Enqvist and P. Olesen, Phys. Lett. B319 (1993) 178.

[45] M. Hindmarsh and A. Everett, astro-ph/9708004.

[46] G.K. Savvidy, Phys. Lett. 71 (1977) 133.

[47] K. Enqvist and P. Olesen Phys. Lett. B329 (1994) 195.

[48] J.M. Cornwall, Phys. Rev. D56 (1997) 6146.

[49] M. Joyce and M. Shaposnikov, astro-ph/9703005.

[50] M. Giovannini and M. Shaposnikov, hep-ph/9710234; Phys. Rev. Lett. 80 (1998) 22.

[51] B. Ratra, Ap. J. 391 (1992) L1.

[52] A-C. Davis and K. Dimopoulos Phys. Rev. D55 (1997) 7398.

[53] T. Vachaspati and A. Vilenkin, Phys. Rev. Lett. 67 (1991) 1057; T. Vachaspati, Phys. Rev. D45 (1992) 3487; D.N. Vollick, Phys. Rev. D48 (1993) 3585.

[54] K. Dimopoulos, hep-ph/9706513; C.J.A.P. Martins and E.P.S. Shellard, astroph/9706287.

[55] D. Lemoine and M. Lemoine, Phys. Rev. D52 (1995) 1955; M. Gasperini, M. Giovannini and G. Veneziano, Phys. Rev. Lett. 75 (1995) 3796.

[56] A. P. Martin and A-C. Davis, Phys. Lett. B360 (1995) 71.

[57] K. Dimopoulos and A-C. Davis Phys. Lett. B390 (1997) 87.

[58] For a review and further references, see T. Bohr, M. H. Jensen, G. Paladin and A. Vulpiani, Dynamical Systems Approach to Turbulence, Cambridge Nonlinear Science Series (Cambridge University Press, 1996).

[59] J. Silk, Ap. J. 151 (1968) 459.

[60] K. Jedamzik, V. Katalinic, and A. Olinto, astro-ph/9606080

[61] P. Olesen, Phys. Lett. B398 (1997) 321. 